\title{
Chromosomal alterations of pediatric malignancy in a West Texas population
}

\author{
Aditya Rajan BS, Sahil Tonk, Chhanda Bose PhD, Sharda P. Singh PhD, \\ Sriman Swarup MD, Tejaswini P Reddy BS, Santosh Chavali BS, Kishore Bhende MD, Philip T. Palade PhD, \\ Ashly Hindle PhD, Meenakshi Darden MD, Vijay Tonk PhD, Sanjay Awasthi MD
}

\begin{abstract}
Background: Comparative genomic hybridization (CGH) microarrays are used for genome-wide evaluation of copy number variations (CNV) of known prognostic significance; however, unannotated variants of uncertain significance (VUS) are frequently present. To identify potentially actionable targets, we retrospectively analyzed VUS loci using CGH data from 192 cases of cancer or genetic disorders treated at TTUHSC.

Methods: DNA was hybridized onto CytoSure Constitution V3 arrays, scanned with the Agilent microarray $D$ scanner, and analyzed by CytoSure Interpret Software.

Results: We found 794 distinct CNVs, the most frequent being 14q32.22 (112 rearrangements), 14q11.2(100), 8p11.2 (98), 15q11.1-q11.2 (83), and 8p23.1 (77). In particular, $8 p 11.22$ alterations were found in many pediatric tumors, with gain/loss ratio of 4.7 . Linkage of TACC1, TM2D2, KAT6A and ADAM32 was indicated by a similar 5-year survival rate of $75.3 \%$ $(n=253)$, which was greater than in unaltered cases $(62.2 \%, n=15,809$ cases) in The Cancer Genome Atlas database.
\end{abstract}

Conclusion: Knockdown of genes occurring at variants of uncertain significance (VUS) loci may help identify new therapy targets.

Keywords: ADAM32, TACC1, KAT6A, TM2D2, 8p11.21

\section{INTRODUCTION}

Anatomist Wilhelm Waldeyer coined the term chromosome ${ }^{1,2}$ for the analine dye-stained intranuclear threads that coalesced and separated during cell division, observed earlier by microscopist Walther Fleming, the founder of cytogenetics. ${ }^{3}$ Though the origin of modern genetics is attributed to the simultaneous rediscovery of Gregor Mendel's classical work

Corresponding author: Sanjay Awasthi; Sharda Singh and Vijay Tonk

Contact Information: Sanjay.Awasthi@ttuhsc.edu; Sharda.Singh@ttuhsc.edu; Vijay.tonk@ttuhsc.edu DOI: 10.12746/swrccc.v8i33.627 by the botanists Hugo DeVries, Carl Correns, and Erich von Tschermak, ${ }^{4}$ the discovery of the role of chromosomes as the microstructural basis of heredity can be attributed to Theodor Boveri, who used sea urchin cells to show that concerted interactions of chromosomes, centrosomes, and centrioles were necessary for transmission of genetic traits. ${ }^{5}$ Progress in cytogenetics was hampered by the physical limits of light microscopy and the vagaries of cytological preparation, as exemplified by the fact that even the number of human chromosomes was not correctly determined until $1956^{6}$ after over 7 decades of estimation. ${ }^{7,8}$ Improvements in microscopy proved critical for the development of the science of cytogenetics, as microscopy provided crucial details on the number, size, shape, and staining patterns of chromosomes. 
Cytogenetic knowledge, combined with the work of numerous biochemists, biophysicists, and molecular biologists, provided evidence for the one-gene one-enzyme theory, ${ }^{9}$ the function of nucleic acids as genetic biomolecules, ${ }^{10}$ elucidation of the structure of DNA, ${ }^{11}$ nucleic acid sequencing methods, ${ }^{12}$ recombinant DNA technology, ${ }^{13}$ microarrays, and massively parallel nucleotide sequencing methods ${ }^{14}$ which comprise modern clinical human genetics, the central driver of the progress of modern medicine to cure diseases.

Present day clinical cytogenetics evolved through standardization of cytological preparation and image processing techniques yielding consistent identification of aberrations based on characteristic alternating light and dark colored stripes that appear when denatured chromosomes are stained with Giemsa stains (G-bands) $^{15}$ or fluorescent stripes when stained with quinacrine (Q-bands) denaturing processes. ${ }^{16}$ Improved cytological methods allowed visualization of chromosomal aberrations associated with numerous hereditary syndromes ${ }^{17}$ including large-scale chromosomal rearrangements, monosomies, and trisomies.

High-resolution cytogenetic analyses have revealed less readily detectable but increasingly identifiable microdeletion and microamplification syndromes, some eponymous such as Zelweger syndrome ${ }^{18}$ and Norrie disease, ${ }^{19}$ and others referred to only by the abnormal chromosomal locus, i.e., microdeletion 8p21.2, microdeletion 8p12p21, microamplification $8 p 23.1$, and over 100 others. $^{20}$

In addition to hereditary disorders recognizable by congenital malformations or biochemical abnormalities, as predicted by Boveri, ${ }^{21}$ the science of cytogenetics has revealed the role of germline as well as somatic chromosomal aberrations in the pathogenesis of cancer. ${ }^{22}$ Indeed, each of the above syndromes is associated with rare forms of pediatric and adult malignancies. The first oncogenic somatic chromosomal aberration identified was the Philadelphia chromosome in chronic myelogenous leukemia, leading to a revolutionary treatment that inhibits the kinase encoded at the $t(9 ; 22)(q 34 ; q 11)$ locus. ${ }^{23}$ Since then, chromosomal aberrations have been established as driver events, prognosticators, or predictors of therapeutic responses for multiple types of malignancies, including many hematologic cancers, ${ }^{24}$ sarcomas, ${ }^{25}$ gliomas, ${ }^{26}$ prostate cancer, ${ }^{27}$ and many others. ${ }^{28}$ Cytogenetic studies have also identified numerous chromosomal loci with putative tumor suppressor genes associated with hereditary cancers or syndromes, including ${ }^{29-31}$ neurofibromatosis, retinoblastoma, ataxia telangiectasia, Cowden's syndrome, Li-Fraumeni syndrome, and others. ${ }^{32}$

The advent of fluorescence in situ hybridization (FISH), a test that detects copy number variations (CNV) based on annealing of fluorescent nucleotide probes to chromosomes, has greatly increased the power of clinical cytogenetic testing. ${ }^{33,34}$ For example, detection of ERBB2 gene amplification is the clinical standard that determines whether breast cancer can be treated with anti-ERBB2 antibodies trastuzumab and pertuzumab. ${ }^{35}$ In addition, several multiprobe FISH assays are broadly used clinically to detect amplification or deletion of genes, guiding prognostic as well as predictive biomarkers in a wide spectrum of malignancies including myeloid and lymphoid leukemia, ${ }^{36,37}$ lymphoma, ${ }^{38,39}$ multiple myeloma, ${ }^{40}$ melanoma, ${ }^{41}$ sarcoma, ${ }^{42}$ and lung cancer. ${ }^{43}$

Despite its clinical utility, FISH does have several limitations including false positivity due to occasional problems in detection or in interpretation of signal patterns in interphase nuclei, ${ }^{44}$ inability to detect some inter-chromosomal rearrangements, ${ }^{45}$ and inability to detect rearrangements under approximately $300 \mathrm{~kb} .{ }^{46}$ It is very useful clinically for confirming suspected copy number variations for loci already recognized as pathogenic, or as classifiers, predictors, prognosticators, or biomarkers for classification, but it is not wellsuited for discovery of new potentially pathogenic genes.

Comparative genomic hybridization $(\mathrm{CGH}) \mathrm{mi}-$ croarrays allow simultaneous detection of thousands of CNVs across the entire genome. Fluorescencelabeled total genomic DNA of the test sample is hybridized to metaphase chromosomes or DNA microarrays, quantified using array scanners, and normalized to a reference DNA sample, i.e., non-cancerous tissue. The intensity ratio correlates with $\mathrm{CNVs}$ in regions as short as a few thousand or even a few hundred bases. ${ }^{47,48}$ Technical complexities that can 
yield false positives or negatives have been largely overcome through stringent validation, multiple array elements, and computational methods such that clinical application is now quite feasible and widely used. Though the aim is to detect pathogenic CNVs with clinically significant implications, many $\mathrm{CNV}$ s have been observed that can only be classified as variants of uncertain significance (VUS), which pose significant challenges ${ }^{49,50}$ but also the opportunity to discover novel pathogenic mechanisms in cancer and other hereditary diseases.

Toward this goal, we catalogued CNVs observed in our West Texas pediatric (as well as some adult) oncology patients, identified CNVs present at unexpectedly high frequencies, and evaluated potential oncogenic roles of amplified genes by assessing the effects of gene knockdown on the growth of human cancer cell lines. We found a very high frequency of 8p11.2 amplification, with 4 genes (TACC1, TM2D2, KAT6A and ADAM32) residing at the locus.

\section{MATERIALS AND METHODS}

The Maxwell 16 LEV blood DNA kit and Maxwell 16 instrument were used to extract the DNA from peripheral blood leukocytes, bone marrow aspirates, and tumor samples. The Maxwell 16 MDx (Promega, Madison, WI, USA) cartridge was used for DNA purification, and NanoDrop 1000 spectrophotometer (Thermo Fisher Scientific, Waltham, MA, USA) was used for estimation of purity. Purified slides were hybridized onto the CytoSure Constitution V3 array (8x60K) for 24 hours, scanned with the Agilent microarray D scanner, and evaluated for CNVs, $\mathrm{LOH}$ and uniparental disomy with the included CytoSure Interpret Software. All methods meet CAP/CLIA requirements. Software was updated using the Deciphering Developmental Disorders (DDD) study and the latest updates from ClinGen, the Clinical Genome Resource. Where applicable, conventional cytogenetics and FISH assays were used to validate results. Data were de-identified and no identifying registry was created. The data were sorted by karyotype, microarray results of gain or loss, diagnosis, gender, and age.

\section{RESULTS}

Our aim was to determine the overall frequency of each chromosomal aberration and to discover novel disease associations. The sample population of the study consisted of 192 individuals, 166 of whom were pediatric patients. The ages ranged from 1 month to 70 years, with the median age being 10.1 and 20.7 years for the pediatric and adult patients, respectively. The male/female ratio was 1.1 and 1.2 for the pediatric and adult patients, respectively. Final histopathological diagnosis was unavailable for 28 cases, identified on test requisitions only by suspected diagnosis, clinical reason to perform the test, or site of biopsy. While all 192 patients were included in calculating the relative frequency of chromosomal alterations, cases without definitive pathological diagnosis were excluded from disease-specific analyses.

B-and T-cell ALL or lymphoma were analyzed as an aggregate, with the vast majority $(>90 \%)$ being B-cell. Due to multiple histological types of sarcoma, they were also analyzed as an aggregate (Suppl. Table 1). As expected in this primarily pediatric population, the most common diagnosis was ALL (51 cases, 2 adults, 29 male), followed by lymphoma (12 cases, 4 adults, 9 males), Wilm's tumor ( 9 cases, 4 adults, 5 males), neuroblastoma ( 9 cases, 0 adults, 4 males), and sarcoma (35 cases, 4 adults, 22 males). Other malignant diagnoses included AML (4 cases, one adult, 0 males), hepatocellular carcinoma (2 cases, 0 adult, 1 male with fibrolamellar histology), and melanoma ( 2 cases, 1 adult, 1 male). The remaining cases had benign diagnoses such as hemangioma, dysmorphic features, congenital pulmonary airway malformation, eosinophilia, enchondroma, cerebellar AV malformation, craniosynostosis, undescended testes, desmoid tumor, suspected Beckwith-Weidemann syndrome, and thyroid nodule.

\section{FREQUENTLY ALTERED CHROMOSOMAL LOCI}

We found 794 unique chromosomal loci. The ratio of gains to losses was 1.04 . At loci with gains, $11.2 \%$ involved mosaic gains and $5.0 \%$ had two copy gains; at loci with losses, mosaic loss was found in $10.0 \%$ and two-copy loss was present in $4.0 \%$. Among the 
rearrangements, $51.3 \%$ were known pathogenic, $20.7 \%$ were variants of uncertain significance, and $28 \%$ were known to be non-pathogenic. Among pathogenic variants, the most common locus with germline alterations was Xp22.33q28, which had pathogenic rearrangements in 16 cases $(2.3 \%$ of pathogenic alterations), with 12 of these $(75 \%)$ being gains. $14 q 11.2$ and $4 p 16.3$ were the next most frequently involved loci. 4p16.3 was involved in 13 cases of pathogenic mutations $(1.9 \%)$ and was found to have loss of genetic material in 7 of these cases (53.8\%). Due to the relatively low frequency of alterations at these sites, we decided to focus on the cases with the largest number of alterations.

\section{FREQUENTLY ALTERED CHROMOSOMAL LOCI BY DISEASE}

Consult Table 1 and Table 2 for summaries of the CNV loci, patient ages, and sex ratios further described below. Among patients diagnosed with ALL, the top altered loci were $14 q 32.33$ (38 cases), 14q11.2 (29 cases), 8p11.2 (20 cases), 8p23.1 (19 cases), 2p11.2 (12 cases) and 15q11.1q11.2 (12 cases) (Suppl. Table 2 ).
The $14 q 32.33$ locus, the location of the immunoglobulin heavy $(I G H)$ gene, is subject to VDJ recombination. Monoclonal rearrangements are associated with $B$ cell neoplasms while polyclonal ones are benign. ${ }^{51}$ While the $14 q 11.2$ and $2 \mathrm{p} 11.2$ loci have previously been implicated in the pathology of ALL, ${ }^{52} 8 p 23.1$ and 8p11.2 have not previously been reported in ALL. ${ }^{53}$ Among patients with sarcoma, the top altered loci were 8p23.1 (18 cases), 14q32.33 (18 cases), 8p11.22 (16 cases), and 14q11.2 (14 cases) (Suppl. Table 3). All loci have been previously reported in osteosarcoma, ${ }^{54}$ Ewing's sarcoma, rhabdomyosarcoma, and pleomorphic high-grade sarcoma. ${ }^{55}$ Male predominance was noted for 14q11.2 (10:4).

Among lymphoma patients, the top altered loci were $14 q 32.33$ ( 8 cases), 8p11.22 (7 cases), 14q11.2 (6 cases), and 8p23.1 (6 cases) (Suppl. Table 4). ${ }^{56}$ $8 p 23.1$ and $14 q 11.2$ have been known to play a role in cancer in large B cell lymphoma and T cell lymphoma respectively, while $8 p 11.22$ has not been reported previously in lymphoma. ${ }^{57}$

Among patients diagnosed with Wilm's tumor, the top three altered loci were 14q32.33 (5 cases),

Table 1. Altered chromosomal loci by disease

\begin{tabular}{|l|l|l|l|}
\hline DX (no. of cases) & Age Range & Male:Female & Most common Chr Locus altered \\
\hline ALL (51) & $1.58-69.55$ & $29: 22$ & $14 \mathrm{q} 32.33,14 \mathrm{q} 11.2,8 \mathrm{p} 11.22,8 \mathrm{p} 23.1$ \\
\hline Wilm's tumor (9) & $1.04-22.75$ & $4: 5$ & $14 \mathrm{q} 32.33,8 \mathrm{p} 11.22,8 \mathrm{p} 23.2$ \\
\hline Neuroblastoma (9) & $2.11-4.68$ & $5: 4$ & $14 \mathrm{q} 32.33,8 \mathrm{p} 23.1,10 \mathrm{q} 26.3,14 \mathrm{q} 11.2,8 \mathrm{p} 11.22$ \\
\hline Sarcoma (13) & $3.68-24.69$ & $6: 5$ & $8 \mathrm{p} 23.1,14 \mathrm{q} 11.2,10 \mathrm{q} 26.3,14 \mathrm{q} 32.33$ \\
\hline Lymphoma (12) & $9.94-39.95$ & $9: 3$ & $14 \mathrm{q} 32.33,8 \mathrm{p} 11.22,14 \mathrm{q} 11.2,8 \mathrm{p} 23.1$ \\
\hline Bone Tumor (7) & $10.1-16.09$ & $6: 2$ & $14 \mathrm{q} 32.33,8 \mathrm{p} 11.22,8 \mathrm{p} 23.1,14 \mathrm{q} 11.2$ \\
\hline Fibromatosis (5) & $17.14-21.09$ & $5: 0$ & $17 \mathrm{q} 12,8 \mathrm{p} 23.1$ \\
\hline Breast Mass (5) & $3.05-18.84$ & $0: 5$ & $8 \mathrm{p} 11.22,14 \mathrm{q} 32.33,15 \mathrm{q} 11.2$ \\
\hline AML (4) & $11: 85-44: 78$ & $0: 4$ & $14 \mathrm{q} 11.2$ \\
\hline Testicular Tumor (3) & $16.4-20.07$ & $3: 0$ & $12 \mathrm{p} 13.33 \mathrm{p} 11.1,10 \mathrm{p} 15.3 \mathrm{q} 26.3,14 \mathrm{q} 32.33,19 \mathrm{p} 13.3 \mathrm{q} 13.34$ \\
\hline Hepatocellular Cancer (2) & $15.86-16.4$ & $1: 1$ & $8 \mathrm{p} 23.1,16 \mathrm{q} 22.1,2 \mathrm{p} 11.2$ \\
\hline Melanoma (2) & $4.47-37.29$ & $1: 1$ & $14 \mathrm{q} 11.2,14 \mathrm{q} 32.33$ \\
\hline Oral Lesion (2) & $9.88-10.49$ & $2: 0$ & $14 \mathrm{q} 11.2,14 \mathrm{q} 32.33,15 \mathrm{q} 11.2,3 \mathrm{p} 26.3$ \\
\hline
\end{tabular}

Each diagnosis is listed by age range, sex ratio and the most common chromosomal loci affected. Diagnoses with less than 2 cases are excluded. Chr - chromosome; AML - acute myelogenous leukemia. 
Table 2. Previously unreported association of cancers with chromosomal loci

\begin{tabular}{|l|l|l|}
\hline DX & Known chromosomal loci & Previously unreported chromosomal loci \\
\hline ALL & $14 \mathrm{q} 11.2,2 \mathrm{p} 11.2$ & $8 \mathrm{p} 11.22,8 \mathrm{p} 23.1$ \\
\hline Wilm's tumor & $2 \mathrm{q} 37$ & $8 \mathrm{p} 11.22,8 \mathrm{p} 23.1,14 \mathrm{q} 11.2,16 \mathrm{q} 11.2 \mathrm{q} 24.3,1 \mathrm{q} 21.1 \mathrm{q} 44,2 \mathrm{q} 13$ \\
\hline Neuroblastoma & $14 \mathrm{q} 11.2$ & $8 \mathrm{p} 23.1,8 \mathrm{p} 11.22,10 \mathrm{q} 26.13$ \\
\hline Sarcoma & $8 \mathrm{p} 23.1,14 \mathrm{q} 11.2,10 \mathrm{q} 26.13$ & none \\
\hline Lymphoma & $8 \mathrm{p} 11.22,14 \mathrm{q} 11.2$ & $8 \mathrm{p} 23.1$ \\
\hline Fibromatosis & none & $17 \mathrm{q} 12,8 \mathrm{p} 11.22,8 \mathrm{p} 23.1$ \\
\hline AML & $14 \mathrm{q} 11.2$ & none \\
\hline
\end{tabular}

AML - acute myelogenous leukemia.

8p11.22 (4 cases), and 8p23.2 (4 cases) (Suppl. Table 5). 8p23.2 was also found with enchondroma (1 case) and fibromatosis (1 case). We found three males with 1q21.1q44 and three females with $2 q 13$. None of the 5 loci mentioned have been previously reported in Wilm's tumor cases.

Among patients diagnosed with neuroblastoma, the top altered loci were $14 q 32.33$ (6 cases), 8p23.1 (6 cases), 10q26.13 (5 cases), 14q11.2 (5 cases), and 8p11.22 (5 cases) (Suppl. Table 6). None of these loci except $14 q 11.2$ has been reported in neuroblastoma. ${ }^{58}$ Male predominance was noted for 8p23.1 (5:1) and 10q26.13 (4:1).

Across these cancers, the five most commonly affected chromosomal loci were $14 q 32.33$ (112 cases), 14q11.2 (100 cases), 8p11.2 (98 cases), $15 q 11.1-15 q 11.2$ (83 cases), and 8p23.1 (77 cases). At the $8 p 11.2$ locus, gains (80 cases) far outnumbered losses (18 cases) (Suppl. Table 7). At the 15q11.1$15 q 11.2$ locus, losses (54 cases) outnumbered gains (29 cases) (Suppl. Table 8). At the 8p23.1 locus losses (62 cases) also outnumbered gains (15 cases) (Suppl. Table 9). Sixty-two probes, many overlapping, covered the $14 q 32.33$ locus, with gains as well as losses observed within cases. The two most common regions affected in patients were rs106535904-rs106561150 (56 cases with 22 gains and 34 losses) (Supp. Table 1) and rs106686490-rs106824658 (52 cases with 10 gains and 32 losses) (Suppl. Table 1). The region $14 q 32.33$ is home to the Ig heavy chain locus and subject to VDJ recombination; the significance of its presence here is unclear. Seventy probes covered the $14 q 11.2$ region, with both gains and losses also observed within cases. The most commonly affected regions here were rs22481496-rs22974566 (14q11.2) (56 cases, 50 gains and 6 losses) (Suppl. Table 10) and rs20203456-rs20331146 (14q11.2) (23 cases, 4 gains and 19 losses) (Suppl. Table 11).

\section{LOCI KNOWN TO OCCUR IN MICRODELETION OR MICRODUPLICATION SYNDROMES}

In recent years, a number of microdeletion and microduplication syndromes have been associated with specific rearrangements at several chromosomal loci. ${ }^{59-63}$ These are often associated with dysmorphic features or other phenotypes but have not been specifically linked to malignancies. Some of the most common loci in our study (14q11.2, 8p11.2, $8 p 23.1,10 q 26.13$, and 17q12) are involved in these syndromes. Clinical features commonly involve facial abnormalities, developmental delay, intellectual disability, and cardiac defects, although unique features include precocious puberty in 8p11.2; inverted duplication, cryptorchidism, and diaphragmatic hernia in 8p23.1 deletion syndrome, and maturity onset diabetes of the young in $17 q 12$ deletion syndrome. A potential association of these syndromes with cancer has not been previously reported.

\section{Genes of INTEREST AT 8P11.2}

Of all loci, 8p11.2 was of interest because of its high frequency and known involvement in cancer. ${ }^{64-69}$ 
This locus has been of interest in breast ${ }^{65}$ and hepatocellular carcinoma, ${ }^{64}$ and evidence suggests that specific genes at this locus are altered in colorectal, prostate, gastric and renal cell cancer. ${ }^{68}$ There have been multiple oncogenes associated with this site, including FGFR1 at 8p11.23. ${ }^{69}$ There have also been multiple cancer-related fusion proteins and translocations identified involving this site. ${ }^{66}$ The high ratio of gains to losses (80 vs. 18) in our study would support the possibility of multiple oncogenes at this locus.

The three segments of $8 p 11.2$ involved in our study (8p11.21, 8p11.22, 8p11.23) have all been reported to contain several genes that contribute to the development of cancer (Figure 1). These include KAT6A, TACC1, TM2D2, and ADAM32. ${ }^{67,70}$ Notably, 84 out of 98 patients had 8p11.22 rearrangements
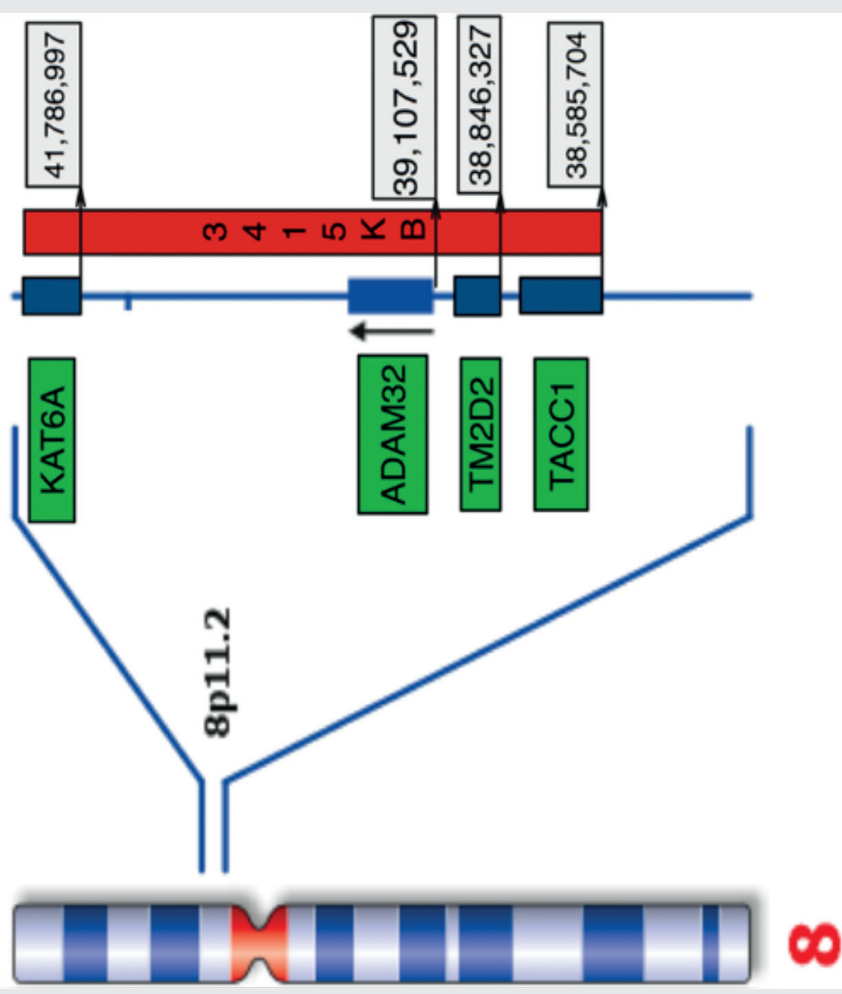

in a segment containing $A D A M 32$ and pseudogene $A D A M 3 A .{ }^{30} A D A M 32$ has previously been found to be elevated in breast, colorectal, gastric, ovarian, prostate, renal, lung, and pediatric medulloblastoma ${ }^{68}$ as well as hepatocellular carcinoma. ${ }^{64}$

We found $A D A M 32$ was gained in acute lymphoblastic leukemia, neuroblastoma, fibromatosis, Wilm's tumor, osteosarcoma, rhabdomyosarcoma, lymphoma, testicular cancer, and adenomatous rectal polyps, malignancies in which this association has not been previously reported. We reasoned that if this locus contained oncogenes, survival of patients with copy number gains would be worsened, and if tumor suppressors were present, survival could be improved. We queried TCGA (The Cancer Genome Atlas) using cBioportal to determine the overall effect of alterations of the four genes in all malignancies $(n=15,809){ }^{71}$

Figure 1. Cytogenetic locations of ADAM32, TACC1, KATA6 and TM2D2.

The ADAM32 (Metallopeptidase Domain 32) gene encodes a disintegrin family membrane-anchored protein involved in brain development, fertilization, tumor development and inflammation. It is located in a cluster of other disintegrin and metallopeptidase family genes on chromosome 8. Alternative splicing results in multiple transcript variants encoding different isoforms. TACC1 (Transforming Acidic Coiled-Coil Containing Protein 1) is a candidate breast cancer gene located near FGFR1 at $8 \mathrm{p} 11.8$ that is amplified in some breast cancers. It is possibly involved in promoting cell division prior tissue differentiation. KAT6A (Lysine Acetyltransferase 6A) is a member of the MOZ, YBFR2, SAS2, and TIP60 family of histone acetyltransferases. It is a part of a complex that acetylates lysine- 9 residues in histone 3 . Chromosomal translocations of this gene have been associated with acute myeloid leukemia. Alternative splicing results in multiple transcript variants. TM2D2 (Beta-Amyloid-Binding Protein-Like Protein 1) encodes a structural module related to the seven transmembrane domain G protein-coupled receptor superfamily. This protein may have regulatory roles in cell death or proliferation signal cascades. This gene has multiple alternatively spliced transcript variants which encode two different isoforms. 


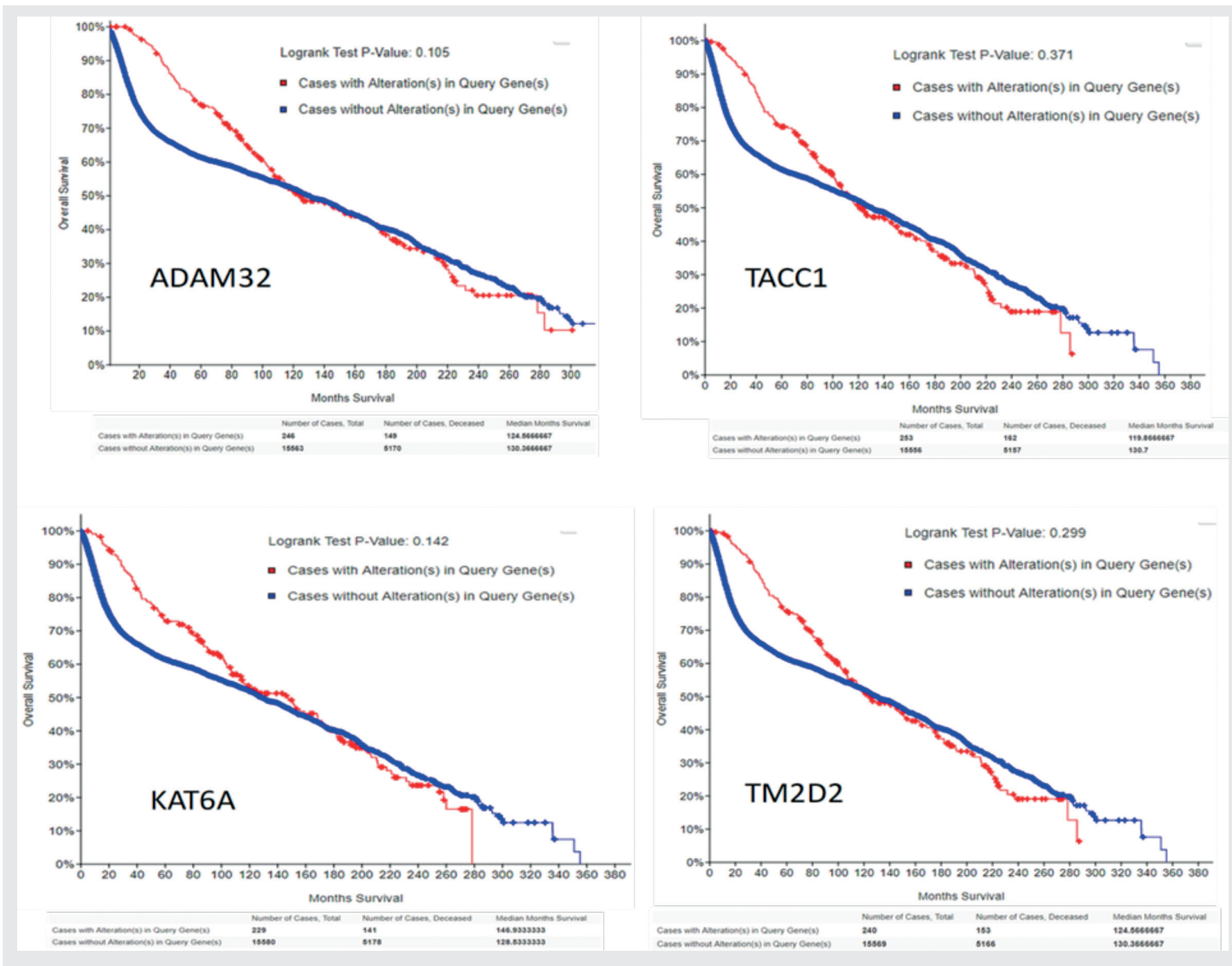

Figure 2. Overall survival across all malignancies according to gene alteration in TCGA.

We found that the percentage of survivors at 5 years was greater in all cases in which any gene was altered as compared with cases without alterations (Figure 2). Interestingly, the percent of survivors $(75.3+1.7 \%)$ was nearly the same regardless of which gene was queried, suggesting co-occurrence of alterations, as would transpire if the amplified locus spanned all four genes in the majority of altered cases. Overall, gains significantly outnumbered losses across all cancers, except for the fibrolamellar variant of hepatocellular carcinoma where all altered cases were losses
(Figure 3). The similarity of histograms of alterations across cancers for all four genes also supported strong linkage (Figure 3 ). Though relatively little is known of the function of TACC1, it appears to participate in the mitotic spindle complex and is possibly regulated by Aurora kinases (Figure 4). ${ }^{72,73}$

\section{Discussion}

Our study is the first retrospective data analysis on microarray data for cancer patients in the West 

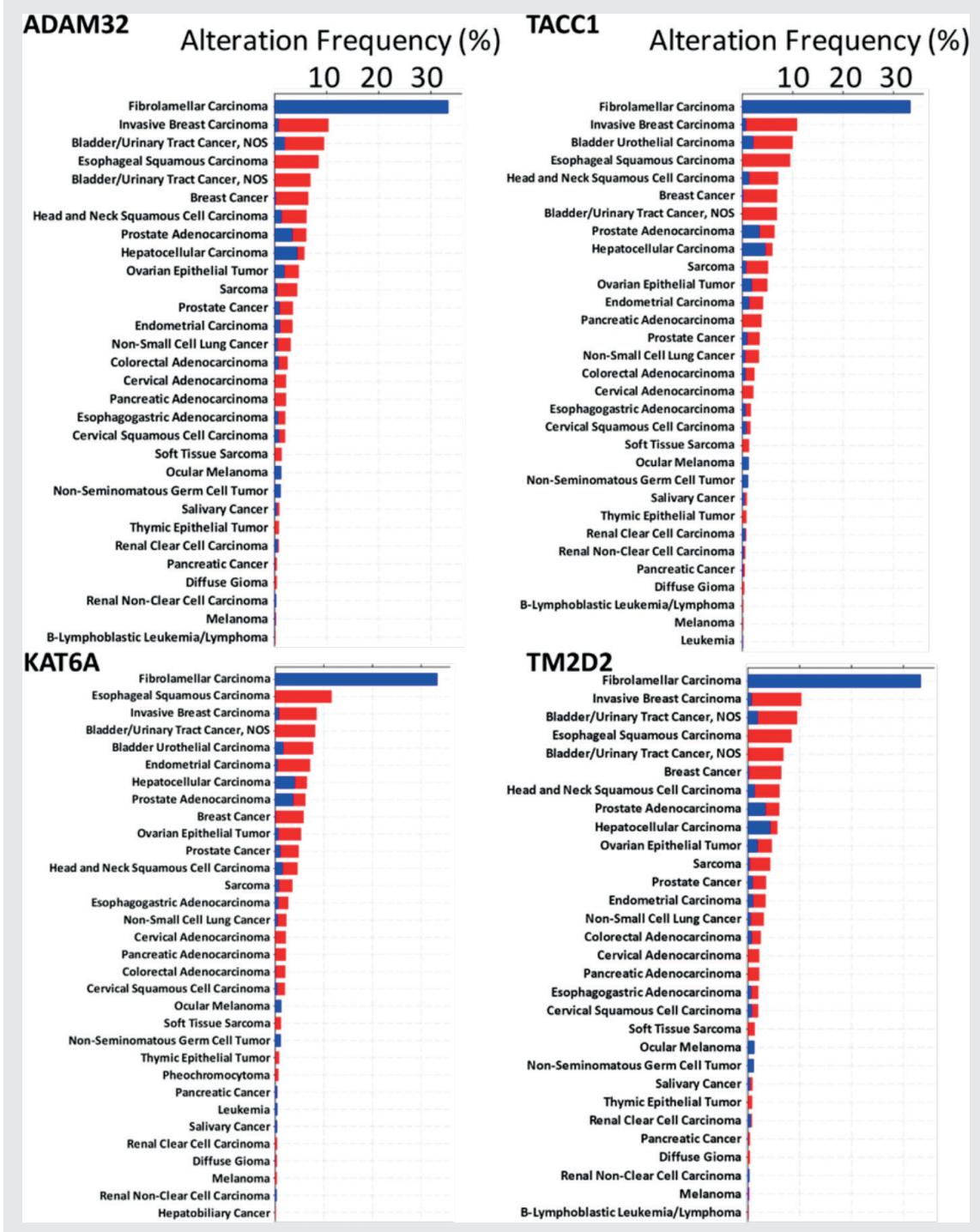

Figure 3. Frequency of CNVs in $A D A M 32$, $K A T 6 A, T A C C 1$ and $T M 2 D 2$ according to TCGA subset of curated non-redundant cases (41,192 in 155 studies). Results are presented by cancer type and sorted by frequency. Red indicates copy number gains and blue indicates copy number losses. The vast majority of cases in TCGA are from adults.

Texas region, and the results showed a number of findings of interest that have not been reported previously. In our primarily pediatric patient population with the main diagnoses of ALL, Iymphoma, sarcoma, Wilm's tumor and neuroblastoma, the five most common rearrangements were at loci $14 \mathrm{q} 11.2$, 14q32.33, 15q11.1-15q11.2, 8p23.1, and 8p11.2. The two rearrangements with the most discernible patterns of expression were $8 \mathrm{p} 23.1$ and $8 \mathrm{p} 11.2$. The $8 p 11.2$ locus has been previously reported to play a role in cancer and had narrowest range of chromosomal coordinates, allowing us to identify affected genes with greater precision. Though survival data from TCGA suggested that the four genes at this locus - ADAM32, TACC1, TM2D2 and KAT6A should be tumor suppressors, we found that knockdown of individual genes actually inhibited growth; the exception was growth stimulation only in melanoma following $A D A M 32$ knockdown, a finding possibly related to complex effects on cancer cell survival with regard to multiple gene amplifications in humans. Knockdown studies confer value by allowing individual genes to be validated as possible cancer therapy targets. 


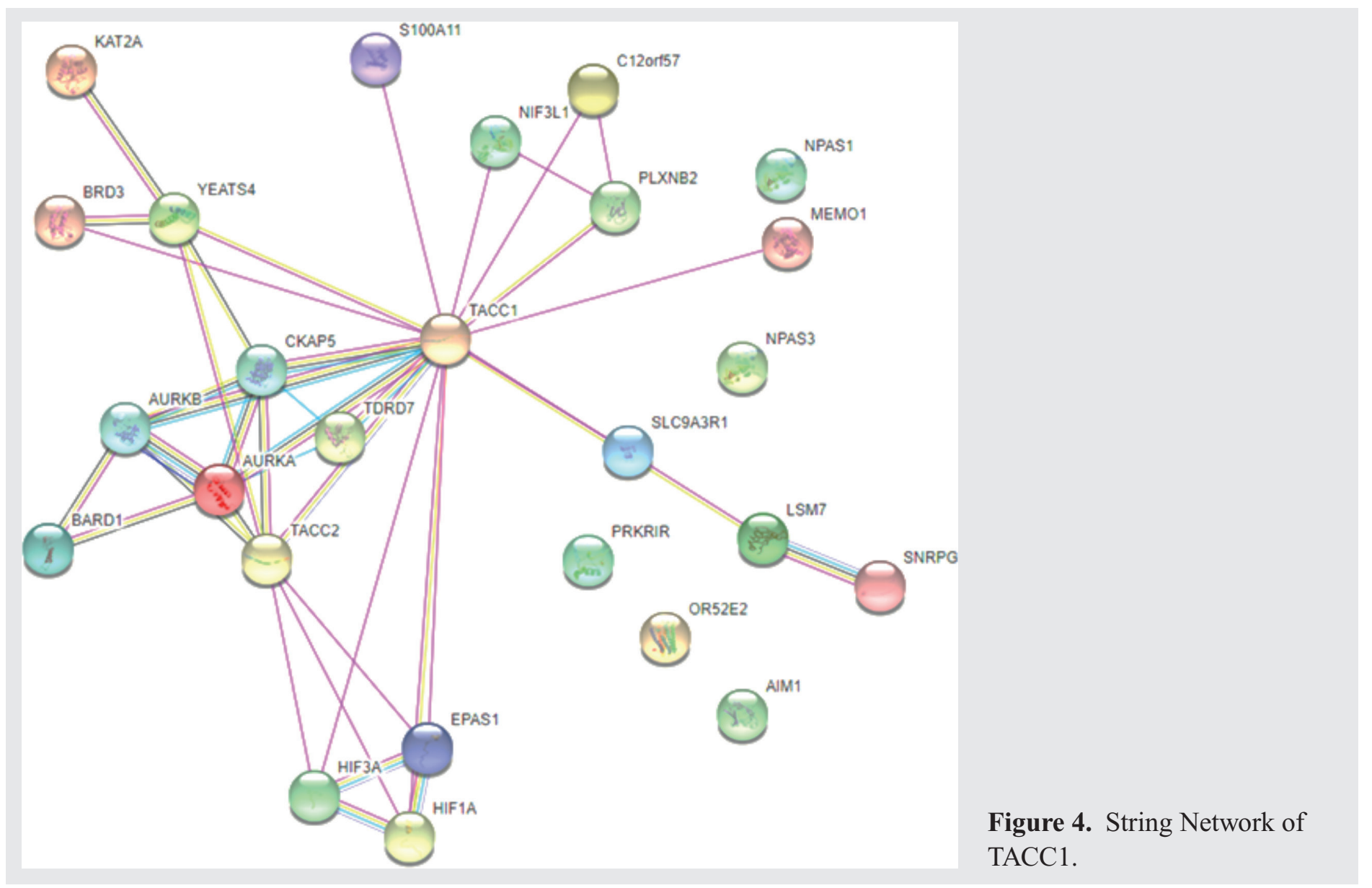

Ours is the first report focused specifically on the patient population of West Texas, an ethnically diverse cohort with exposure to significant environmental factors including extremely iron-rich soil contaminated with numerous herbicides such as carcinogenic glyphosate, pesticides such as endosulfan, and hydrocarbon pollutants from extensive oil and natural gas drilling. ${ }^{74}$ Furthermore, the region may be impacted by the founder effect, as the population has remained relatively isolated.

This novel information has the potential to expand knowledge on the effects of microdeletion and microduplication syndromes as the most common loci in our study (14q11.2, 8p11.2, 8p23.1, 10q26.13, and 17q12) are involved in many syndromes, the clinical features of which primarily center around developmental anomalies. $14 q 11.2$ is involved in both duplication and deletion syndromes, both of which involve facial anomalies such as hypertelorism. Duplication of $14 q 11.2$ also involves ataxia, stereotypical hand movements, hypotonia, and cleft palate..$^{59} 8 \mathrm{p} 11.2$ is involved in both inverted duplication and deletion syndromes, the former of which is more likely to be present in our patient population. ${ }^{60}$ Clinical features of inverted duplication include developmental delays in speech and language, prominent forehead with inverted nostrils, short neck, precocious puberty, heart defects, abnormally increased height, initial hypotonia with developing hypertonia, spastic quadriplegia, and contracted joints in adulthood. Duplication and deletion syndromes have also been described for $8 p 23.1$, the deletion syndrome, which matches the data in our study, involves low birth weight, cardiac defects, diaphragmatic hernias, and 
cryptorchidism. ${ }^{61} 17 q 12$ is more commonly involved in deletion syndromes, although duplication syndromes have also been described and are marked by kidney failure, abnormal behavioral development, and mental retardation. ${ }^{62} 10 \mathrm{q} 26.13$ is involved in the deletion syndrome distal monosomy $10 \mathrm{q} \cdot{ }^{63}$ Clinical features of this syndrome include abnormal facial features, cardiac defects, and ambiguous genitalia, though cancer susceptibility has not yet been described.

The $8 p 11.2$ locus has been recognized to have a broad importance in cancer, and thus our study focuses on four genes of interest positioned at this locus: TACC1, KAT6A TM2D2 and ADAM32 [3]. Deletions and alternative splicing of TACC1 have been associated with an increased risk of gastric cancer, ovarian cancer, breast cancer, and glioblastoma. ${ }^{72,75,76}$ In particular, a TACC1-FGFR fusion protein has been associated with the pathogenesis of glioblastoma multiforme in a subset of patients. ${ }^{72}$ Abnormalities in KAT6A are associated with $A M L$ and breast cancer $(105,106)$. KAT6A-TIF2 and KAT6A-CBP fusions are cytogenetic markers in AML. ${ }^{77}$ Analysis of a breast cancer cell line also showed elevated levels of KAT6A. ${ }^{78}$

ADAM32 is a member of the ADAM family of genes that encodes cell surface proteins involved in inflammation, tumor formation, fertilization, and brain development. ${ }^{64,79}$ ADAM32 encodes a protein possessing three functional domains: a disintegrin domain, a metalloproteinase domain, and an epidermal growth factor-like domain. The best-known role of ADAM32 is in the sperm-egg interaction and sperm development, where it is expressed during meiotic prophase in pachytene spermatocytes. ${ }^{80}$ While there have been limited studies on the involvement of $A D A M 32$ in cancer, three prior studies, including a published pediatric pan-cancer genome analysis, have yielded significant data supporting its role in malignancy. One study detects the presence of ADAM32 fusions in ERBB2-amplified breast cancer. ${ }^{65}$ A second showed that $A D A M 32$ was upregulated in hepatocellular cancer. ${ }^{64}$ Genomic rearrangements in ADAM32 were also demonstrated in eight different tumor types including breast, colorectal, gastric, ovarian, prostrate, renal, and lung cancer, as well as pediatric medulloblastoma. ${ }^{68}$
The 8p23.1 locus has a multitude of known oncogenes, such as those encoding the $\alpha$ - and $\beta$-defensin proteins, as well as zinc finger domain proteins and ubiquitin signal peptidase family genes. ${ }^{81-87}$ The $\alpha$-defensins are known to inhibit growth of CML and lung adenocarcinoma cells, though at lower levels they may increase growth of renal cell carcinoma. ${ }^{81-83}$ The $\beta$-defensins suppress tumor invasion and migration as exemplified by beta defensin-1 (DEFB1) effects on oral squamous carcinoma. ${ }^{82}$ SNPs in the $\beta$-defensin genes are associated with increased risk of developing glioblastoma, ${ }^{84}$ while DEFB1 has been shown to have a protective role against cancer and is downregulated in HCV-infected hepatocellular cancer. $^{86}$ Several genes in the 8p23.1 locus encode for USP17-like proteins, which have been found to suppress tumorigenesis in breast cancer. ${ }^{87}$ In our study, 8p23.1 showed deletions in ALL, Wilm's tumor, neuroblastoma, lymphoma and fibromatosis, which has not been reported previously.

Our study is limited by the fact that a DNA microarray can only detect CNVs and is not sensitive to translocations and inversions. Our study is also limited by sample size in the case of several types of cancer including hepatocellular cancer, medulloblastoma, rhabdomyosarcoma, renal cell carcinoma, ovarian tumor, melanoma and peripheral neural epithelioma, in which fewer than 3 cases each were recorded. Finally, it remains possible that many of the chromosomal aberrations described are secondary events without a driver function in cancer, though further evidence is needed to comprehensively elucidate the significance of these rearrangements.

\section{ACKNOWLedgements}

This study was supported by research funds provided to SA and AR by Dr. Steven Berk, Dean, Texas Tech University Health Sciences Center. Additional funding was provided by the Ethel $S$. Neely and Emma S. Treadwell Endowment to SA and the Department of Defense grant BC171159 to SA. We also acknowledge the support of the Clinical Research Institute of the Texas Tech University Health Sciences Center. 
Article citation: Rajan A, Tonk S, Bose C, Singh SP, Swarup S, Reddy TP, Chavali S, Bhende K, Palade PT, Hindle A, Darden M, Tonk V, Awasthi S. Chromosomal alterations of pediatric malignancy in a West Texas population. The Southwest Respiratory and Critical Care Chronicles 2020;8(33):7-20

From: Departments of Internal Medicine (AR, ST, CB, SPS, SS, AH, SA) and Pediatrics (TPR, SC, KB, VT), Texas Tech University Health Sciences Center, Lubbock, Texas; Department of Pharmacology and Toxicology (PTP), University of Arkansas for Medical Sciences, Little Rock, AR; McGovern Medical School of the University of Texas Health Sciences Center (MD), Houston TX.

Submitted: $1 / 7 / 2020$

Accepted: 1/21/2020

Reviewer: Kenneth Nugent MD

Conflicts of interest: none

This work is licensed under a Creative Commons

Attribution-ShareAlike 4.0 International License.

\section{REFERENCES}

1. Waldeyer W. Über Karyokinese und ihre Beziehungen zu den Befruchtungsvorgängen. Bonn: Cohen; 1888.

2. Scheuerlein $H$, Henschke F, Kockerling F. Wilhelm von Waldeyer-Hartz-a great forefather: his contributions to anatomy with particular attention to "his" fascia. Front Surg 2017;4:74. Epub 2017/12/20. doi: 10.3389/fsurg.2017.00074.

3. Flemming W. Zellsubstanz, Kern und Zelltheilung. Flemming W, editor. Leipzig: Vogel; 1882, p. 1-462.

4. Zirkle C. The role of Liberty Hyde Bailey and Hugo de Vries in the rediscovery of Mendelism. J History Biology 1968;1(2): $205-18$.

5. Boveri T. Die Befruchtung und Teilung des Eies von Ascaris megalocephala. Boveri T, editor. Jena: Fischer; 1888.

6. Tjio JH, Levan A. The chromosome number of man. HRD2 Hereditas 1956;42(1-2):1-6.

7. Arnold J. Beobachtungen über Kerntheilungen in den Zellen der Geschwülste. Archiv für Pathologische Anatomie und Physiologie und für Klinische Medicin 1879;78(2):279-301. doi: 10.1007/bf01878412.

8. Painter TS. The Y-chromosome in mammals. Science 1921; 53(1378):503-4. Epub 1921/05/27. doi: 10.1126/science.53. 1378.503 .
9. Hickman M, Cairns J. The centenary of the one-gene one-enzyme hypothesis. Genetics 2003;163(3):839-41. Epub 2003/ $03 / 29$.

10. Hershey AD, Chase M. Independent functions of viral protein and nucleic acid in growth of bacteriophage. Selected papers on virology. 1964.

11. Watson JD, Crick F. Molecular structure of nucleic acids: a structure for deoxyribose nucleic acid. Classic papers in genetics. 1959.

12. Sanger F, Nicklen $S$, Coulson AR. DNA sequencing with chain-terminating inhibitors. Proc Natl Acad Sci U S A 1977;74(12):5463-7. Epub 1977/12/01. doi: 10.1073/pnas. 74.12 .5463 .

13. Arber W, Linn S. DNA modification and restriction. Annu Rev Biochem 1969;38:467-500. Epub 1969/01/01. doi: 10.1146/ annurev.bi.38.070169.002343.

14. Bumgarner R. Overview of DNA microarrays: types, applications, and their future. Current protocols in molecular biology, 2013; Chapter 22. Epub 2013/01/05. doi: 10.1002/ 0471142727.mb2201s101.

15. Seabright $\mathrm{M}$. A rapid banding technique for human chromosomes. Lancet 1971;2(7731):971-2. Epub 1971/10/30. doi: 10.1016/s0140-6736(71)90287-x.

16. Caspersson T, Zech L, Johansson C. Differential binding of alkylating fluorochromes in human chromosomes. Exp Cell Res 1970;60(3):315-9. Epub 1970/06/01. doi: 10.1016/ 0014-4827(70)90523-9.

17. Tommerup N. Mendelian cytogenetics. Chromosome rearrangements associated with mendelian disorders. J Med Genetics 1993;30(9):713-27.Epub 1993/09/01. doi: 10.1136/ jmg.30.9.713.

18. Naritomi K, Hyakuna N, Suzuki Y, et al. Zellweger syndrome and a microdeletion of the proximal long arm of chromosome 7. Human genetics 1988;80(2):201-2. Epub 1988/10/01. doi: 10.1007/bf00702873.

19. Collins FA, Murphy DL, Reiss AL, et al. Clinical, biochemi$\mathrm{cal}$, and neuropsychiatric evaluation of a patient with a contiguous gene syndrome due to a microdeletion Xp11.3 including the Norrie disease locus and monoamine oxidase (MAOA and $M A O B)$ genes. Am J Med Genetics 1992;42(1):127-34.

20. Weise A, Mrasek K, Klein E, et al. Microdeletion and microduplication syndromes. J Histochemistry Cytochemistry 2012;60(5):346-58. Epub 2012/03/08. doi: 10.1369/ 0022155412440001.

21. Boveri T. Zur Frage der Entstehung maligner Tumoren mit 2 Abb. Jena: Fischer; 1914.

22. Frohling S, Dohner H. Chromosomal abnormalities in cancer. N Engl J Med 2008;359(7):722-34.

23. Deininger M, Buchdunger E, Druker BJ. The development of imatinib as a therapeutic agent for chronic myeloid leukemia. 
Blood 2005;105(7):2640-53. doi: 10.1182/blood-2004-083097.

24. Yang JJ, Park TS, Wan TS. Recurrent cytogenetic abnormalities in acute myeloid leukemia. Methods Mol Biol 2017;1541:223-45. Epub 2016/12/03. doi: 10.1007/978-14939-6703-2_19.

25. Helman LJ, Meltzer P. Mechanisms of sarcoma development. Nature Rev Cancer 2003;3(9):685-94. Epub 2003/09/03. doi: $10.1038 / \mathrm{nrc} 1168$.

26. Beroukhim R, Getz G, Nghiemphu L, et al. Assessing the significance of chromosomal aberrations in cancer: methodology and application to glioma. Proc Natl Acad Sci U S A 2007;104(50):20007-12. Epub 2007/12/14. doi: 10.1073/ pnas.0710052104.

27. Kluth M, Ahrary R, Hube-Magg C, et al. Genomic deletion of chromosome $12 p$ is an independent prognostic marker in prostate cancer. Oncotarget 2015;6(29):27966-79.

28. Wan TSK. Cancer cytogenetics: methods and protocols. Wan TSK, editor. New York, NY: Humana Press; 2017.

29. Weinberg RA. Tumor suppressor genes. Science 1991; 254(5035):1138-46. Epub 1991/11/22. doi: 10.1126/science. 1659741.

30. Tommerup N, Lothe R. Constitutional ring chromosomes and tumor suppressor genes. J Med Genetics 1992;29(12):87982. doi: DOI 10.1136/jmg.29.12.879.

31. Sandberg AA. Translocations in malignant tumors. Am J Pathol 2001;159(6):1979-80. Epub 2001/12/06. doi: 10.1016/ S0002-9440(10)63047-7.

32. Mulvihill JJ. Catalog of human cancer genes : McKusick's Mendelian inheritance in man for clinical and research oncologists (onco-MIM). Baltimore: Johns Hopkins University Press; 1999.

33. Jeffrey ML, Robert HS. Fluorescence in situ hybridization: past, present and future. J Cell Sci 2003;116(14); 2833-8.

34. Gall JG, Pardue ML. Formation and detection of RNA-DNA hybrid molecules in cytological preparations. Proc Natl Acad Sci U S A 1969;63(2):378-83. Epub 1969/06/01. doi: 10.1073/pnas.63.2.378.

35. Carney WP, Leitzel K, Ali S, et al. HER-2 therapy. HER-2/ neu diagnostics in breast cancer. Breast Cancer Research 2007;9(3):1-11.

36. Avet-Loiseau K. FISH analysis at diagnosis in acute lymphoblastic leukemia. Leukemia Lymphoma 1999;33(5-6):4419. doi: Doi 10.3109/10428199909058449.

37. Anna V, Diana B, Alistair GR, et al. FISH mapping of Philadelphia negative BCR/ABL1 positive CML. Mol Cytogenetics 2008; $1: 14$.

38. Ventura RA, Martin-Subero JI, Jones M, et al. FISH analysis for the detection of lymphoma-associated chromosomal abnormalities in routine paraffin-embedded tissue. J Mol Diagn 2006;8(2):141-51. Epub 2006/04/29. doi: 10.2353/ jmoldx.2006.050083.

39. Miles RR, Shah RK, Frazer JK. Molecular genetics of childhood, adolescent and young adult non-Hodgkin lymphoma. Br J Haematol 2016;173(4):582-96. Epub 2016/03/13. doi: 10.1111/bjh.14011.

40. Ma ES, Wang CL, Wong AT, et al. Target fluorescence in-situ hybridization (Target FISH) for plasma cell enrichment in myeloma. Mol Cytogenet 2016;9(1):63. Epub 2016/08/18. doi: 10.1186/s13039-016-0263-7.

41. Kansal AR, Shaul AJ, Stern S, et al. Cost-effectiveness of a FISH assay for the diagnosis of melanoma in the USA. Expert Rev Pharmacoecon Outcomes Res 2013;13(3):37180. Epub 2013/05/16. doi: 10.1586/erp.13.22.

42. Asif A, Mushtaq S, Hassan U, et al. Fluorescence in situ hybridization (FISH) for differential diagnosis of soft tissue sarcomas. Asian Pacific J Cancer Prevention 2018;19(3): 655-60. Epub 2018/03/28. doi: 10.22034/APJCP.2018.19.3. 655 .

43. Vollbrecht $\mathrm{C}$, Lenze $\mathrm{D}$, Hummel M, et al. RNA-based analysis of ALK fusions in non-small cell lung cancer cases showing IHC/FISH discordance. BMC Cancer 2018;18(1):1158. Epub 2018/11/24. doi: 10.1186/s12885-018-5070-6.

44. Braha E, Martiniuc V, Panzaru M, et al. Prenatal diagnosis of gonosomal anomalies: limitations of the FISH method and genetic counseling difficulties in 15 cases. Rev Med Chir Soc Med Nat Iasi 2013;117(2):450-6. Epub 2013/ $12 / 18$.

45. White SJ, Sterrenburg E, van Ommen GJB, et al. An alternative to FISH: detecting deletion and duplication carriers within 24 hours. J Med Genetics 2003;40(10). doi: ARTN e113 DOI 10.1136/jmg.40.10.e113.

46. Sun MR, Zhang H, Li GY, et al. Molecular characterization of 20 small supernumerary marker chromosome cases using array comparative genomic hybridization and fluorescence in situ hybridization. Scientific Reports 2017;7(1). doi: ARTN 10395 10.1038/s41598-017-10466-z.

47. Kallioniemi A, Kallioniemi OP, Sudar D, et al. Comparative genomic hybridization for molecular cytogenetic analysis of solid tumors. Science 1992;258(5083):818-21. Epub 1992/10/30. doi: 10.1126/science.1359641.

48. Pinkel D, Albertson DG. Array comparative genomic hybridization and its applications in cancer. Nat Genet 2005;37 Suppl:S11-7. Epub 2005/05/28. doi: 10.1038/ng1569.

49. Dharmadhikari AV, Ghosh R, Yuan B, et al. Copy number variant and runs of homozygosity detection by microarrays enabled more precise molecular diagnoses in 11,020 clinical exome cases. Genome Med 2019;11(1):30. Epub 2019/05/19. doi: 10.1186/s13073-019-0639-5. 
50. Nowakowska B. Clinical interpretation of copy number variants in the human genome. J Appl Genet 2017;58(4):44957. Epub 2017/10/01. doi: 10.1007/s13353-017-0407-4.

51. Bagg A, Braziel RM, Arber DA, et al. Immunoglobulin heavy chain gene analysis in lymphomas: a multi-center study demonstrating the heterogeneity of performance of polymerase chain reaction assays. J Mol Diagn 2002;4(2):81-9. Epub 2002/05/03. doi: 10.1016/S1525-1578(10)60685-X.

52. Studd JB, Yang MJ, Li ZH, et al. Genetic predisposition to B-cell acute lymphoblastic leukemia at 14q11.2 is mediated by a CEBPE promoter polymorphism. Leukemia 2019; 33(1):1-14. doi: 10.1038/s41375-018-0184-z.

53. Steinemann D, Cario G, Stanulla $M$, et al. Copy number alterations in childhood acute lymphoblastic leukemia and their association with minimal residual disease. Gene Chromosome Canc 2008;47(6):471-80. doi: 10.1002/gcc.20557.

54. dos Santos Aguiar S, de Jesus Girotto Zambaldi L, dos Santos AM, et al. Comparative genomic hybridization analysis of abnormalities in chromosome 21 in childhood osteosarcoma. Cancer Genet Cytogenet 2007;175(1):35-40. Epub 2007/05/15. doi: 10.1016/j.cancergencyto.2007.01.007.

55. Liu $\mathrm{C}$, Li D, Hu J, et al. Chromosomal and genetic imbalances in Chinese patients with rhabdomyosarcoma detected by high-resolution array comparative genomic hybridization. Intern J Clin Experimental Path 2014;7(2):690-8.

56. Scandurra M, Mian M, Greiner TC, et al. Genomic lesions associated with a different clinical outcome in diffuse large B-Cell lymphoma treated with R-CHOP-21. Br J Haematol 2010;151(3):221-31. Epub 2010/09/04. doi: 10.1111/j.13652141.2010.08326.x.

57. $\mathrm{Ng} \mathrm{SB}$, Chung $\mathrm{TH}$, Kato $\mathrm{S}$, et al. Epstein-Barr virusassociated primary nodal T/NK-cell lymphoma shows a distinct molecular signature and copy number changes. Haematologica 2018;103(2):278-87. doi: 10.3324/haematol. 2017.180430.

58. Ho N, Peng H, Mayoh $\mathrm{C}$, et al. Delineation of the frequency and boundary of chromosomal copy number variations in paediatric neuroblastoma. Cell Cycle 2018;17(6):749-58. Epub 2018/01/23. doi: 10.1080/15384101.2017.1421875.

59. Delvallez NM. Orphanet: 14q11.2 microdeletion syndrome 2011. Available from: https://www.orpha.net/consor/cgi-bin/ OC_Exp.php?lng=EN\&Expert=261120.

60. Fryns J-P. Orphanet: $8 \mathrm{p}$ inverted duplication/deletion syndrome 2013. Available from: https://www.orpha.net/consor/ cgi-bin/OC_Exp.php?lng=EN\&Expert=96092.

61. Devriendt K. Orphanet: $8 \mathrm{p} 23.1$ microdeletion syndrome 2012. Available from: https:/www.orpha.net/consor/cgi-bin/ OC_Exp.php?lng=en\&Expert=251071.

62. Mitchell MW, De-Luca DM, Martin CL. Orphanet: $17 \mathrm{q} 12$ microdeletion syndrome 2016. Available from: https://www.
orpha.net/consor/cgi-bin/OC_Exp.php?Lng=EN\&Expert= 261265.

63. Leheup B. Orphanet: Distal monosomy 10q 2008. Available from: https://www.orpha.net/consor/cgi-bin/OC_Exp.php? lng $=$ EN\&Expert $=96148$.

64. Zou B, Liu X, GongY, et al. A novel 12-marker panel of cancerassociated fibroblasts involved in progression of hepatocellular carcinoma. Cancer Management Res 2018;10: 5303-11. Epub 2018/11/23. doi: 10.2147/CMAR.S176152.

65. Vasmatzis G, Wang X, Smadbeck JB, et al. Chromoanasynthesis is a common mechanism that leads to ERBB2 amplifications in a cohort of early stage HER2(+) breast cancer samples. BMC Cancer 2018;18(1). doi: ARTN 738 10.1186/ s12885-018-4594-0.

66. Singh D, Chan JM, Zoppoli P, et al. Transforming fusions of FGFR and TACC genes in human glioblastoma. Science 2012;337(6099):1231-5. Epub 2012/07/28. doi: 10.1126/ science. 1220834.

67. Robinson JT, Thorvaldsdottir H, Winckler W, et al. Integrative genomics viewer. Nat Biotechnol 2011;29(1):24-6. Epub 2011/01/12. doi: 10.1038/nbt.1754.

68. Marczok S, Bortz B, Wang C, et al. Comprehensive analysis of genome rearrangements in eight human malignant tumor tissues. PloS One 2016;11(7):e0158995. Epub 2016/07/09. doi: 10.1371/journal.pone.0158995.

69. Karajannis MA, Vincent L, Direnzo R, et al. Activation of FGFR1beta signaling pathway promotes survival, migration and resistance to chemotherapy in acute myeloid leukemia cells. Leukemia 2006;20(6):979-86. Epub 2006/04/07. doi: $10.1038 /$ sj.leu.2404203.

70. KAT6A, TACC1, TM2D2, et al. Atlas of Genetics and Cytogenetics in Oncology and Haematology 2018. Available from: http://www.atlasgeneticsoncology.org.

71. Cerami E, Gao J, Dogrusoz U, et al. The cBio cancer genomics portal: an open platform for exploring multidimensional cancer genomics data. Cancer Discov 2012;2(5): 401-4. Epub 2012/05/17. doi: 10.1158/2159-8290.CD-120095.

72. Nathalie C, BÈnÈdicte D, Christophe G, et al. TACC1chTOGAurora A protein complex in breast cancer. Oncogene 2003;22:8102-16.

73. Gergely F, Karlsson C, Still I, et al. The TACC domain identifies a family of centrosomal proteins that can interact with microtubules. Proc Natl Acad Sci USA 2000;97(26):143527. doi: DOI 10.1073/pnas.97.26.14352.

74. Collier K, Hopkins J S, Leven R. As oil and gas exports surge, West Texas becomes the world's "extraction colony." The Texas Tribune. 2018.

75. Lauffart B, Vaughan MM, Eddy R, et al. Aberrations of TACC1 and TACC3 are associated with ovarian cancer. BMC 
Womens Health 2005;5:8. Epub 2005/05/28. doi: 10.1186/ 1472-6874-5-8.

76. Line A, Slucka Z, Stengrevics A, et al. Altered splicing pattern of TACC1 mRNA in gastric cancer. Cancer Genet Cytogenet 2002;139(1):78-83. Epub 2003/01/28. doi: 10.1016/s01654608(02)00607-6.

77. Deguchi K, Ayton PM, Carapeti M, et al. MOZ-TIF2induced acute myeloid leukemia requires the MOZ nucleosome binding motif and TIF2-mediated recruitment of CBP. Cancer Cell 2003;3(3):259-71. Epub 2003/04/05.

78. Turner-Ivey B, Guest ST, Irish JC, et al. KAT6A, a Chromatin modifier from the 8p11-p12 amplicon is a candidate oncogene in luminal breast cancer. Neoplasia 2014;16(8): 644-55. doi: 10.1016/j.neo.2014.07.007.

79. Choi I, Woo JM, Hong S, et al. Identification and characterization of ADAM32 with testis-predominant gene expression. Gene 2003;304:151-62. Epub 2003/02/06. doi: 10.1016/ s0378-1119(02)01202-7.

80. Torabi F, Bogle OA, Estanyol JM, et al. Zona pellucidabinding protein 2 (ZPBP2) and several proteins containing BX7B motifs in human sperm may have hyaluronic acid binding or recognition properties. Mol Hum Reprod 2017; 23(12):803-16. doi: 10.1093/molehr/gax053.

81. Droin N, Jacquel A, Hendra JB, et al. Alpha-defensins secreted by dysplastic granulocytes inhibit the differentiation of monocytes in chronic myelomonocytic leukemia. Blood 2010;115(1):78-88. Epub 2009/10/30. doi: 10.1182/blood2009-05-224352.
82. Han $\mathrm{Q}$, Wang RN, Sun CK, et al. Human beta-defensin-1 suppresses tumor migration and invasion and is an independent predictor for survival of oral squamous cell carcinoma patients. PloS One. 2014;9(3). doi: ARTN e91867 10.1371/journal.pone.0091867. PubMed PMID: WOS: 000333355300042.

83. M,ller CA, Markovic-Lipkovski J, Klatt T, et al. Human alpha-defensins HNPs-1, -2 , and -3 in renal cell carcinoma: influences on tumor cell proliferation. Amer J Path 2002; 160(4):1311-24.

84. Rajaraman $\mathrm{P}$, Brenner AV, Butler MA, et al. Common variation in genes related to innate immunity and risk of adult glioma. Cancer epidemiology, biomarkers prevention 2009;18(5): 1651-8. Epub 2009/05/09. doi: 10.1158/1055-9965.EPI-081041.

85. Xu N, Wang YS, Pan WB, et al. Human alpha-defensin-1 inhibits growth of human lung adenocarcinoma xenograft in nude mice. Molr Cancer Therapeutics 2008;7(6):158897. Epub 2008/06/21. doi: 10.1158/1535-7163.MCT-080010.

86. Ling Y-M, Chen J-Y, Guo L, et al. b-defensin 1 expression in $\mathrm{HCV}$ infected liver/liver cancer: an important role in protecting HCV progression and liver cancer development. Sci Rep 2017;7(1).

87. Chen X, Wang C, Liao KM, et al. USP17 suppresses tumorigenesis and tumor growth through deubiquitinating AEP. Intern J Biol Sci 2019;15(4):738-48. doi: 10.7150/ ijbs.30106. 